\section{Genotypic Variation of Total Phenol and Oleuropein Concentration and Antioxidant Activity of 11 Greek Olive Cultivars (Olea europaea L.)}

\author{
Antonios Petridis ${ }^{1}$ and Ioannis Therios \\ Department of Horticulture, Laboratory of Pomology, Aristotle University of \\ Thessaloniki, 54124 Thessaloniki, Greece
}

Georgios Samouris

Laboratory of Hygiene and Technology of Food of Animal Origin, Veterinary Research Institute of Thessaloniki, National Agricultural Research Foundation (NAGREF), 57001 Thermi-Thessaloniki, Greece

Additional index words. olive, phenolic compounds, maturity index

\begin{abstract}
The chemical and biochemical composition of olives relies on some agronomical factors, one of which is the cultivar. In this study, fruits and leaves of 11 Greek olive cultivars were examined concerning their phenol and oleuropein concentrations. Fruit antioxidant activity was determined as well. The obtained results showed that significant differences existed among cultivars regardless of the tissue or the measured parameter. In general, leaves had higher total phenol and oleuropein concentrations than fruits. Finally, the highest oleuropein concentration in fruits was recorded in 'Pikrolia Kerkiras' followed by 'Romeiki', 'Megaritiki', 'Kothreiki', and 'Kalamon'. These cultivars may constitute the raw material in the industrial production of oleuropein.
\end{abstract}

The Mediterranean Sea basin is the main olive-producing region all over the world. Greece constitutes one of the most important countries in olive culture, accounting for $13 \%$ and $5.3 \%$ of the global olive oil and table olive production, respectively (International Olive Oil Council, 2011). There are $\approx 60$ olive tree cultivars originating from Greece, some of which are cultivated globally (i.e., 'Koroneiki', 'Kalamon'), whereas most of them are found in local areas.

Phenolic compounds are secondary metabolites with a great structural diversity and a wide phylogenetic distribution (Harborne, 1989). Phenolic compounds influence the organoleptic properties of olive fruits and virgin olive oils and are important markers for studying fruit characteristics of different cultivars and controlling olive production processes. Their antioxidant effect is correlated to the high stability of virgin olive oil against heat oxidation and autoxidation processes overtime (Romani et al., 1999). Besides fruits, some studies considered the phenol fraction present in olive leaves (Briante et al., 2002b; Ranalli et al., 2006). Olive leaves are endowed with the most potent free radical scavenging

\footnotetext{
Received for publication 3 Jan. 2012. Accepted for publication 30 Jan. 2012.

We are most grateful to the Veterinary Research Institute of Thessaloniki (NAGREF), which provided us with the high-performance liquid chromatography unit and had been accommodating us during our experimentation.

${ }^{1}$ To whom reprint requests should be addressed; e-mail antonispet@hotmail.com.
}

power among the different parts of olive trees and provide considerable amounts of high added-value compounds (Japón-Luján et al., 2006). Olive leaves have phenol composition similar to that of olive fruit with oleuropein constituting the main phenolic compound followed by other phenol glycosides (BenaventeGarcía et al., 2000).

Oleuropein is a heterosidic ester of $\beta$-glucosylated elenolic acid and hydroxytyrosol and can be easily transformed by endogenous or exogenous supply of the enzyme $\beta$-glucosidase into glucose and oleuropein aglycon (Ranalli et al., 2006). It is also the most abundant phenolic compound in olive leaves and fruits and is considered as the main responsible factor for the characteristic bitterness of olive fruit and the browning that occurs in green table olives either after impact and wounding during harvesting or during subsequent technological treatments (Malik and Bradford, 2006; Soler-Rivas et al., 2000; Therios, 2009). Health benefits of oleuropein have been extensively investigated. Specifically, it has been reported that oleuropein and other related phenolic compounds such as hydroxytyrosol, tyrosol, verbascoside, ligustroside, and demethyloleuropein act as antioxidants that contribute to a lower risk of coronary diseases (Beauchamp et al., 2005; Visioli et al., 1998; Wiseman et al., 1996) and several types of cancers (Owen et al., 2000; Tripoli et al., 2005). Furthermore, these compounds present antimicrobial and antiviral activity (Bisingnano et al., 1999; Uccella, 2001).

The chemical and biochemical composition of olives relies on some agronomical factors, one of which is the cultivar. Because phenolic compounds play an important role in olive fruit and virgin olive oil quality and present several health benefits, the aim of the present study was to determine the phenolic content and fruit antioxidant activity of 11 Greek olive cultivars. This is of great importance to explore phenolic concentration of the previously mentioned cultivars and identify those with high values to use them commercially for oleuropein production.

\section{Materials and Methods}

Experimental olive orchard. The study was carried out in a field planted with 11 Greek olive cultivars, namely 'Kalamon', 'Kothreiki', 'Maronia', 'Megaritiki', 'Petrolia', 'Pikrolia Kerkiras', 'Romeiki', 'Smertolia', 'Thassitiki', 'Throubolia Aegean', and 'Valanolia' (seven trees per cultivar and of the same age and vegetative habits), during the 2009-2010 season. These 10-year-old olive cultivars were grown in the same orchard located in Poros Troizinia, Northeast Peloponnese, Greece (lat. $37^{\circ} 31^{\prime} \mathrm{N}$, long. $23^{\circ} 29^{\prime}$ E). The climate of the area is Mediterranean with mild winters $\left(9-10^{\circ} \mathrm{C}\right)$ and dry, hot summers $\left(27-28^{\circ} \mathrm{C}\right)$. The mean annual precipitation of the area is $550 \mathrm{~mm}$. The olive plants were subjected to the same cultural conditions (pruning, fertilization, irrigation, and harvesting). For each cultivar, a 3-kg composite sample of fruits and $1 \mathrm{~kg}$ of current-season leaves were collected from all the orientations of the tree canopy on 15 Nov. Each composite sample (fruit or leaf) was divided into three subsamples of $200 \mathrm{~g}$ each.

Maturity index. The maturity index of each cultivar during sampling was determined according to the Spanish National Institute of Agronomic Research (Morelló et al., 2004).

Sample preparation and extraction of phenolic compounds. Fully expanded leaves and fruits from all the orientations of the tree canopy were sampled. All samples were transferred to the laboratory and washed with distilled water. Leaf subsamples (200 g) without the petiole were ground into a fine powder, whereas fruit subsamples were depitted before blending. An aliquot of $250 \mathrm{mg}$ from each tissue (leaf or paste) was extracted in $10 \mathrm{~mL}$ of $80 \%$ methanol on a shaker at $200 \mathrm{rpm}$ for $30 \mathrm{~min}$ at room temperature $\left(23{ }^{\circ} \mathrm{C}\right)$. The mixture was filtered and the procedure was repeated. The two extracts were combined and an aliquot of $2 \mathrm{~mL}$ was transferred to polypropylene screw cap vials and stored at $-80{ }^{\circ} \mathrm{C}$ until high-performance liquid chromatography analysis. Before analyses, the extracts were filtered through a $20-\mu \mathrm{m}$ polytetrafluoroethylene syringe filter.

Total phenol concentration. The total phenol concentration was determined spectrophotometrically at $760 \mathrm{~nm}$ using the FolinCiocalteu reagent (Škerget et al., 2005). A portion of $125 \mu \mathrm{L}$ extract was combined with $2.5 \mathrm{~mL}$ of Folin-Ciocalteu reagent (diluted 10 times with water), $375 \mu \mathrm{L}$ distilled water, and $2 \mathrm{~mL}$ sodium carbonate $\left(75 \mathrm{~g} \cdot \mathrm{L}^{-1}\right)$. The mixture was incubated in a water bath for $5 \mathrm{~min}$ at $50{ }^{\circ} \mathrm{C}$ and after cooling at room temperature 
$\left(23{ }^{\circ} \mathrm{C}\right)$, the optical density at $760 \mathrm{~nm}$ was measured with a spectrophotometer (Camspec M106 spectrophotometer, Leeds, U.K.). Gallic acid at concentrations of $12,28,40$, and 56 $\mu \mathrm{g} \cdot \mathrm{mL}^{-1}$ was used to develop standard curves.

High-performance liquid chromatography analysis of oleuropein. High-performance liquid chromatography (HPLC) analyses were performed using a Perkin Elmer HPLC system (Series 200) equipped with a Perkin Elmer LC-200 diode array detector. Oleuropein was separated by reversed-phase HPLC using a Spherisorb ODS-2 $(250 \mathrm{~mm} \times 4.6 \mathrm{~mm}$ i.d., $5 \mu \mathrm{m}$ particle size; MZ-Analysentechnik GMBH, Mainz, Germany) column maintained at $35{ }^{\circ} \mathrm{C}$ during chromatographic runs. The column was eluted at a flow rate of $1 \mathrm{~mL} \cdot \mathrm{min}^{-1}$ with a gradient of solvent system comprising water (Solvent A, pH adjusted to 3.1 with phosphoric acid) and acetonitrile (Solvent B). Solvent B was increased linearly from $10 \%$ at zero time to $30 \%$ at $10 \mathrm{~min}$, held isocratically for $4 \mathrm{~min}$, and followed by further linear ramping to $40 \%$ at $19 \mathrm{~min}$. Initial conditions were reached in $10 \mathrm{~min}$. A $20-\mu \mathrm{L}$ aliquot of extract or standard solution was injected for each run and elution profiles were detected at $280 \mathrm{~nm}$. Oleuropein was identified by matching retention time and the ultraviolet spectra of a peak in the extract chromatogram with the peak of standard oleuropein solutions. For quantitative measurements of oleuropein, a five-point calibration curve $\left(R^{2}=0.999, \mathrm{y}=\right.$ $4470.32 \mathrm{x}-2.92$ ) was developed on the basis of the corresponding standard substance. Stock standard solution of oleuropein was prepared in methanol at the $1 \mathrm{mg} \cdot \mathrm{mL}^{-1}$ concentration. Standard solutions of oleuropein were prepared at concentrations of $2.5,5,10,25$, and $50 \mu \mathrm{g} \cdot \mathrm{mL}^{-1}$ by diluting appropriate volumes of the stock standard solution in water.

Ferric reducing antioxidant ability of plasma assay. The ferric reducing antioxidant ability of plasma (FRAP) assay (Benzie and Strain, 1996) was used to evaluate the antioxidant activity of the samples. The FRAP method relies on the reduction of TPTZ (2,4,6-tripyridyl-s-triazine)- $-\mathrm{Fe}^{3+}$ complex to TPTZ-Fe ${ }^{2+}$ form with an intense blue color and absorption maximum at $593 \mathrm{~nm}$. FRAP assay is a nonspecific method and the absorption alterations reflect the total reducing power of all the antioxidant substances found in the test solution. A triplicate of each treatment was analyzed spectrophotometrically. Briefly, a weighted amount of $1 \mathrm{~g}$ of sample was extracted twice with $10 \mathrm{~mL}$ of a solution consisting of $50 \%$ methanol in $1.2 \mathrm{M} \mathrm{HCl}$ $(1: 10 \mathrm{w} / \mathrm{v})$ at $4{ }^{\circ} \mathrm{C}$ for $24-\mathrm{h}$ intervals. For the FRAP assay, a FRAP reagent was prepared by mixing acetate buffer $(0.3 \mathrm{M}, \mathrm{pH} 3.6)$, $10 \mathrm{~mm}$ TPTZ in $40 \mathrm{~mm} \mathrm{HCl}$, and $20 \mathrm{mM} \mathrm{FeCl}_{3}$ at 10:1:1 (v/v/v) ratio. The FRAP reagent $(3 \mathrm{~mL})$ and sample extract $(10 \mu \mathrm{L})$ were added in a test tube, vortexed, and incubated immediately in a water bath at $37^{\circ} \mathrm{C}$ for $4 \mathrm{~min}$. After cooling at room temperature $\left(23^{\circ} \mathrm{C}\right)$, the absorbance was recorded at $593 \mathrm{~nm}$. All solutions were prepared at the day of measurements. An aqueous solution of $100 \mu \mathrm{M}$ ascorbic acid was used for calibration of the instrument. Total antioxidant activity is expressed as $\mu \mathrm{mol}$ ascorbic acid equivalent (AAE)/g fresh weight (FW).

Statistics. The data were subjected to oneway analysis of variance using the SPSS 17.0 for Windows statistical package (SPSS, Chicago, IL). Comparison among the means to determine significant differences $(P \leq 0.05)$ were performed using the Duncan's multiple range test.

\section{Results and Discussion}

In both tissues, significant genotypic differences were recorded among cultivars concerning total phenol (TP) concentration (Fig. 1). Fruit TP concentrations ranged from 8.03 $\mathrm{mg} \cdot \mathrm{g}^{-1} \mathrm{FW}$ to $17.96 \mathrm{mg} \cdot \mathrm{g}^{-1} \mathrm{FW}$. The cultivars Valanolia, Pikrolia Kerkiras, Kalamon, and Romeiki presented the highest TP concentrations, whereas 'Thassitiki', 'Megaritiki', 'Maronia', and 'Petrolia' the lowest ones. The remaining cultivars had medium TP values. These values are almost two times higher than those reported by Bouaziz et al. (2010) for some Tunisian cultivars. Leaf TP concentrations ranged from $20.58 \mathrm{mg} \cdot \mathrm{g}^{-1}$
FW to $34.41 \mathrm{mg} \cdot \mathrm{g}^{-1} \mathrm{FW}$ with 'Smertolia' and 'Kothreiki' presenting the highest concentrations and 'Maronia' and 'Thassitiki' the lowest ones (Fig. 1). These data show that both tissues are important sources of phenols. However, for all cultivars, leaves had higher TP concentration than fruits.

The obtained analytical data concerning oleuropein concentration in Olea europaea L. fruits and leaves are shown in Figure 2. The data confirmed that olive fruits and leaves are oleuropein sources with marked differences among cultivars. Specifically, fruit oleuropein concentrations ranged from $0.25 \mathrm{mg} \cdot \mathrm{g}^{-1} \mathrm{FW}$ to $11.07 \mathrm{mg} \cdot \mathrm{g}^{-1} \mathrm{FW}$. The highest oleuropein concentration in fruits was recorded in 'Pikrolia Kerkiras'. This observation is interesting, justifying the Greek name of this cultivar, because "pikros" means "bitter" and oleuropein is responsible for the bitter taste of olives. Cultivars with high oleuropein concentration, but lower than that of the previously mentioned cultivar, were 'Romeiki', 'Megaritiki', 'Kothreiki', and 'Kalamon', whereas 'Maronia' showed the lowest oleuropein values. The measured concentrations of oleuropein in the studied olive

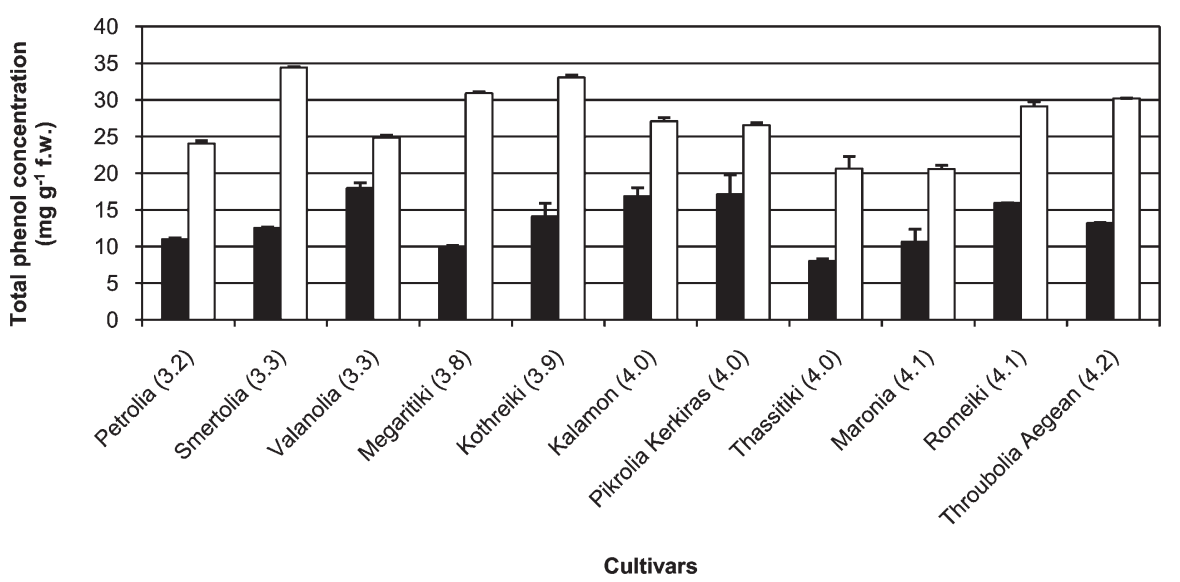

Fig. 1. Total phenol concentration in fruits (black bars) and leaves (white bars) of 11 Greek olive cultivars. Results are expressed as means \pm SE of three determinations. Values into parentheses represent maturity index (MI).

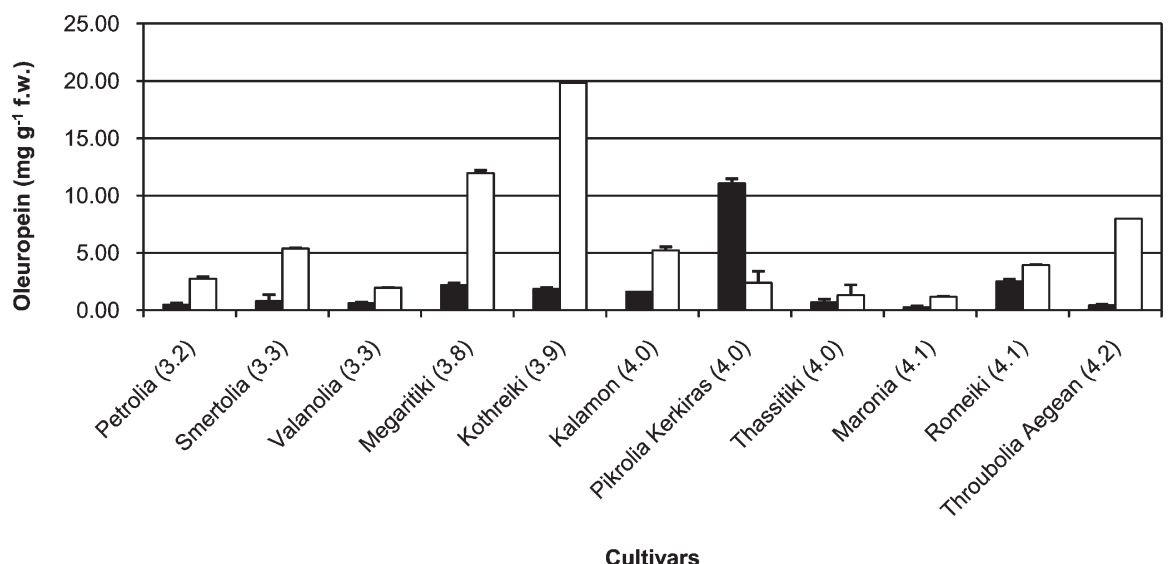

Fig. 2. Oleuropein concentrations in fruits (black bars) and leaves (white bars) of 11 Greek olive cultivars. Results are expressed as means \pm SE of three determinations. Values into parentheses represent maturity index (MI). 


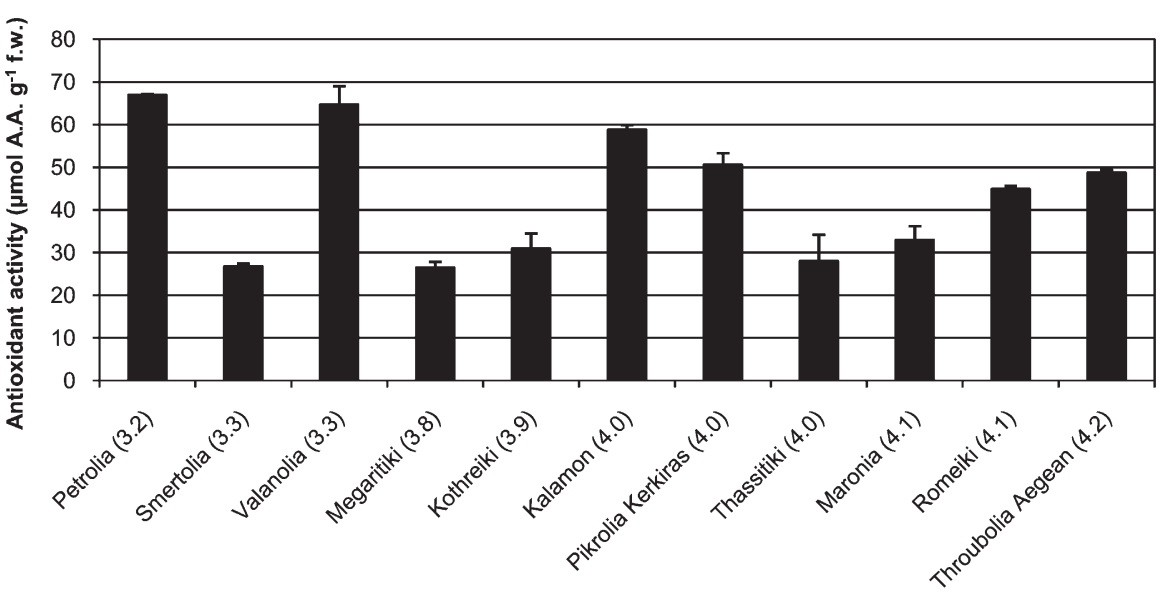

Cultivars

Fig. 3. Fruit antioxidant activity of 11 Greek olive cultivars. Results are expressed as means \pm SE of three determinations. Values into parentheses represent maturity index (MI).

fruits are in general consistent with those reported by other authors (Esti et al., 1998; Ortega-García and Peragón, 2009; Romani et al., 1999; Vigna et al., 2005) for some Italian, Spanish, and Portuguese cultivars with relative differences among them. Oleuropein concentration declines as maturation progresses (Amiot et al., 1989; Briante et al., 2002b; Damak et al., 2008) and relative differences among cultivars could be ascribed to genotype and to their different maturation stages. However, the obtained results clearly show the effect of the genotype on TP and oleuropein concentrations, because the cultivars had similar maturity indices but different concentrations of these compounds. Leaf oleuropein concentration was higher than that of fruits, whereas significant genotypic differences were observed (Fig. 2). The range of leaf oleuropein concentration was $1.16-19.85 \mathrm{mg} \cdot \mathrm{g}^{-1} \mathrm{FW}$. The highest leaf oleuropein concentration was found in 'Kothreiki' followed by 'Megaritiki', 'Throubolia Aegean', and 'Kalamon'. On the other hand, the lowest leaf oleuropein concentration was recorded in 'Maronia', although 'Thassitiki' and 'Valanolia' presented low values as well. Like in the case of fruits, the measured concentrations ranged similarly to those reported by other authors for some Italian and Spanish cultivars (Ortega-García and Peragón, 2009; Ranalli et al., 2006). According to Ranalli et al. (2009), fruits and leaves presented negligible differences in oleuropein concentration, which is in disagreement with our findings. These results show that leaves could be considered as a rich source of oleuropein and proved to be particularly valuable for various technological treatments. Recent examples constitute the mixing of olive leaves ( $2 \%$ to $3 \%$ ) with overripe olives before processing to produce oils with marked flavor, high oleuropein concentration, and high resistance to oxidation (Ranalli et al., 2003, 2004) and the olive leaf extracts in tablet form (Briante et al., 2002a). On this view, the selection of appropriate genotypes, i.e., those possessing high leaf oleuropein concentrations, is of great importance.

Because fruit is the edible part of olive tree, we measured antioxidant activity only in this tissue. Fruit antioxidant activity differed among cultivars as well (Fig. 3). Antioxidant activity ranged from $26.51 \mu \mathrm{mol}$ AAE/g FW to $66.97 \mu \mathrm{mol} \mathrm{AAE} / \mathrm{g} \mathrm{FW}$. The highest antioxidant activity was recorded in 'Petrolia', 'Valanolia', and 'Kalamon'. On the other hand, 'Megaritiki', 'Smertolia', 'Thassitiki', 'Kothreiki', and 'Maronia' had lower antioxidant activity values. In general, antioxidant activity was positively correlated with TP concentration, because cultivars with high TP concentration showed higher antioxidant activity and vice versa.

\section{Literature Cited}

Amiot, M.J., A. Fleuriet, and J.J. Macheix. 1989 Accumulation of oleuropein derivatives during olive maturation. Phytochem. 28:67-69.

Beauchamp, G.K., R.S.J. Keast, D. Morel, J. Lin, J. Pika, Q. Han, C.H. Lee, A.B. Smith, and P.A.S Breslin. 2005. Phytochemistry: Ibuprofen-like activity in extra-virgin olive oil. Nature 437: 45-46.

Benavente-García, O., J. Castillo, J. Lorente, A Ortuño, and J.A. Del Rio. 2000. Antioxidant activity of phenolics extracted from Olea europaea L. leaves. Food Chem. 68:457-462.

Benzie, I. and J. Strain. 1996. The ferric reducing ability of plasma (FRAP) as a measure of 'antioxidant power': The FRAP assay. Anal. Biochem. 239:70-76.

Bisingnano, G., A. Tomaino, R. Lo Cascio, G. Crisafi, N. Uccella, and A. Saija. 1999. On the in vitro antimicrobial activity of oleuropein and hydroxytyrosol. J. Pharm. Pharmacol. 51:971974.

Bouaziz, M., H. Jemai, W. Khabou, and S. Sayadi. 2010. Oil content, phenolic profiling and antioxidant potential of Tunisian olive drupes. J. Sci. Food Agr. 90:1750-1758.

Briante, R., F. Lacara, F. Febbraio, M. Patumi, and R. Nucci. 2002a. Bioactive derivatives from oleuropein by a biotransformation on Olea europaea leaf extracts. J. Biotechnol. 93:109119.
Briante, R., M. Patumi, S. Limongelli, F. Febbraio, C. Vaccaro, A. Di Sale, F. La Cara, and R. Nucci. 2002b. Changes in phenolic and enzymatic activities content during fruit ripening in two Italian cultivars of Olea europaea L. Plant Sci. 162:791-798.

Damak, N., M. Bouaziz, M. Ayadi, S. Sayadi, and M. Damak. 2008. Effect of the maturation process on the phenolic fractions, fatty acids, and antioxidant activity of the Chétoui olive fruit cultivar. J. Agr. Food Chem. 56:15601566.

Esti, M., L. Cinquanta, and E. La Notte. 1998. Phenolic compounds in different olive varieties. J. Agr. Food Chem. 46:32-35.

Harborne, J.B. 1989. General procedures and measurement of total phenolics, p. 2-9. In: Dey, P.M. and J.B. Harborne (eds.). Methods in plant phenolics. Academic Press, London, UK.

International Olive Oil Council. 2011. Olive oil figures. 9 Nov. 2011. <http://www.internationaloliveoil. org/>.

Japón-Luján, R., J. Ruise-Jiménez, and M.D. Luque De Castro. 2006. Discrimination and classification of olive tree varieties and cultivation zones by biophenol contents. J. Agr. Food Chem. 54: 9706-9712.

Malik, N.S.A. and J.M. Bradford. 2006. Changes in oleuropein levels during differentiation and development of floral buds in 'Arbequina' olives. Sci. Hort. 110:274-278.

Morelló, J.R., M.P. Romero, and M.J. Motilva 2004. Effect of the maturation process of the olive fruit on the phenolic fraction of drupes and oils from Arbequina, Farga, and Morrut cultivars. J. Agr. Food Chem. 52:6002-6009.

Ortega-García, F. and J. Peragón. 2009. Phenylalanine ammonia-lyase, polyphenol oxidase, and phenol concentration in fruits of Olea europaea L. cv. Picual, Verdial, Arbequina, and Frantoio during ripening. J. Agr. Food Chem. 57:1033110340.

Owen, R.W., A. Giacosa, W.E. Hull, R. Haubner, B. Spiegelhalder, and H. Bartsch. 2000. The antioxidant/anticancer potential of phenolic compounds isolated from olive oil. Eur. J. Cancer 36:1235-1247.

Ranalli, A., S. Contento, L. Lucera, M. Di Febo, D. Marchegiani, and V. Di Fonzo. 2006. Factors affecting the contents of iridoid oleuropein in olive leaves (Olea europaea L.). J. Agr. Food Chem. 54:434-440.

Ranalli, A., T. Gomes, D. Delcuratolo, S. Contento, and L. Lucera. 2003. Improving virgin olive oil quality by means of innovative extracting biotechnologies. J. Agr. Food Chem. 51:25972602.

Ranalli, A., L. Lucera, S. Contento, N. Simone, and P. Del Re. 2004. Bioactive constituents, flavors and aromas of virgin olive oils obtained by processing olives with a natural enzyme extract. Eur. J. Lipid Sci. Technol. 106:187-197

Ranalli, A., D. Marchegiani, S. Contento, F. Girardi, M.P. Nicolosi, and M.D. Brullo. 2009. Variations of iridoid oleuropein in Italian olive varieties during growth and maturation. Eur. J. Lipid Sci. Technol. 111:678-687.

Romani, A., N. Mulinacci, P. Pinelli, F.F. Vincieri, and A. Cimato. 1999. Polyphenolic content in five Tuscany cultivars of Olea europaea L. J. Agr. Food Chem. 47:964-967.

Škerget, M., P. Kotnik, M. Hadolin, A. Rižner Hraš, M. Simonič, and Ž. Knez. 2005. Phenols, proanthocyanidins, flavones and flavonols in some plant materials and their antioxidant activities. Food Chem. 89:191-198. 
Soler-Rivas, C., J.C. Espín, and H.J. Wichers. 2000. Oleuropein and related compounds. J. Sci. Food Agr. 80:1013-1023.

Therios, I.N. 2009. Olives. Crop Production Science in Horticulture Series. CABI, UK. p. 409.

Tripoli, E., M. Giammanco, G. Tabacchi, D. Di Majo, S. Giammanco, and M. La Guardia. 2005. The phenolic compounds of olive oil: Structure, biological activity and beneficial effects on human health. Nutr. Res. Rev. 18:98-112.
Uccella, N. 2001. Olive biophenols: Biomolecular characterization, distribution and phytoalexin histochemical localization in the drupes. Trends Food Sci. Technol. 11:315-327.

Vigna, A.F., F. Ferreres, B.M. Silva, P. Valentão, A. Gonçalves, J.A. Pereira, M.B. Oliveira, R.M. Seabra, and P.B. Andrade. 2005. Phenolic profiles of Portuguese olive fruits (Olea europaea L.): Influences of cultivar and geographical origin. Food Chem. 89:561-568.
Visioli, S.A., S. Bellosta, and C. Galli. 1998. Oleuropein, the bitter principle of olives, enhances nitric oxide production by mouse macrophages. Life Sci. 62:541-546.

Wiseman, S.A., J. Mathot, N.J. De Fouw, and L.B.M. Tijburg. 1996. Dietary non-tocopherol antioxidants present in extra virgin olive oil increase the resistance of low density lipoproteins to oxidation in rabbits. Atherosclerosis 120:1-2. 